\title{
Feasibility of a Tungsten Rubber Grid Collimator for Electron Grid Therapy
}

\author{
KENTA KIJIMA ${ }^{1}$, ANCHALI KRISANACHINDA ${ }^{2}$, MIKOTO TAMURA ${ }^{1}$, \\ YASUMASA NISHIMURA ${ }^{3}$ and HAJIME MONZEN ${ }^{1}$ \\ ${ }^{1}$ Department of Medical Physics, Graduate School of Medical Sciences, Kindai University, Osaka, Japan; \\ ${ }^{2}$ Department of Radiology, Faculty of Medicine, Chulalongkorn University, Bangkok, Thailand; \\ ${ }^{3}$ Department of Radiation Oncology, Faculty of Medicine, Kindai University, Osaka, Japan
}

\begin{abstract}
Background/Aim: Spatially fractionated radiotherapy (grid therapy) can control some bulky tumors which is challenging for conventional radiotherapy. This study aimed to investigate whether a novel tungsten contained rubber (TCR) grid collimator can be employed in electron grid therapy. Materials and Methods: The TCR grid collimator placed on a solid water phantom, and percentage depth doses (PDDs) and lateral dose profiles were measured for $9 \mathrm{MeV}$ electron beam with Gafchromic EBT3 films. At the lateral dose profile, the ratios of the dose in the areas with and without shielding (valley-to-peak ratios) were evaluated. Results: The $d_{\max }$ values with the 1 , 2 and $3 \mathrm{~mm}$ TCR grid collimators were 1.2, 1.1 and $0.7 \mathrm{~cm}$, respectively, while the valley-to-peak ratios at each $d_{\max }$ were $0.566,0.412$ and 0.293 , respectively. Conclusion: Only the $2 \mathrm{~mm}$ TCR grid collimator had adequate dosimetric features compared to the conventional grid collimator and could be substituted.
\end{abstract}

Spatially fractionated radiotherapy (grid therapy) was introduced by Alban Kohler in 1909 for treating deepseated tumors (1). It was possible to treat without the extensive damage of skin and subcutaneous tissue occurring following irradiation through a special grid collimator with small circular or square apertures. Subsequently, grid therapy was poorly evaluated and hardly ever used until the 1930s. Following the work of Jolles et al. (2) during the orthovoltage era, the usefulness of the grid therapy was shown by Mohiuddin et al. in the

Correspondence to: Hajime Monzen, Ph.D., Department of Medical Physics, Graduate School of Medical Sciences, Kindai University, 377-2, Ohno-Higashi, Osakasayama, Osaka, 859-8511, Japan. Tel: +81 723660221, Fax: +81 723680206, e-mail: hmon@med.kindai.ac.jp

Key Words: Tungsten-contained rubber, electron grid therapy, bulky superficial tumor. late 20th and early 21st century again (3-5). The combination of grid therapy, irradiating a dose of $15 \mathrm{~Gy}$ or higher, and additional external beam resulted in higher response rates than conventional radiotherapy alone. In addition, Ha et al. showed the feasibility of mega-voltage photon grid therapy (4-6), which is able to create a grid of appropriate size and pattern using multileaf collimator. It could treat deep-seated tumors easier than grid therapy using cerrobend (Cerro Metal Products, Bellefonte, PA, USA) grid collimator, although a longer delivery time was needed (7).

Photon grid therapy is an excellent treatment, however electron grid therapy is superior to the photon grid therapy for large and superficial tumors where critical radiosensitive structures are present behind the tumor in the path of the photon beams (7), since the dose of the electron beam rapidly attenuates after passing through a medium (810). Tamura et al. have shown that the Tungsten Functional Paper (TFP) could be used in electron grid therapy, having both radiation shielding ability and paper flexibility properties (11-16), instead of cerrobend by Monte Carlo simulation (10). In a previous study, tungsten-contained rubber (TCR) (B050TE ${ }^{\circledR}$, Hayakawa Rubber Co., Ltd. Hiroshima, Japan) was developed and displayed adequate shielding efficiency against electron beams (17). The cerrobend has odors and is possibly toxic to the human body, due to the vapor cloud of heavy metals during processing and may have harmful effects on the environment (18-20). On the other hand, TCR is a nontoxic, shielding material that is good for the human body and environment due to its ecofriendly products (lead free), has high density and is waterproof $(16,17)$. In addition, owing to its flexibility, TCR can adapt and acquire various conformations such as shoulder and belly (17).

The aim of this study was to show the feasibility of TCR grid collimators in electron grid therapy by a phantom study. The dosimetric characteristics and their possible clinical applications were investigated. 
Table I. Physical properties of tungsten-contained rubber collimator.

\begin{tabular}{lc}
\hline Item & Value \\
\hline Length $(\mathrm{cm})$ & 25 \\
Width $(\mathrm{cm})$ & 25 \\
Density $\left(\mathrm{g} / \mathrm{cm}^{3}\right)$ & $7.65 \pm 0.03$ \\
Hardness (Type-A) & 77 \\
Tensile strength (MPa) & 4.5 \\
Elongation (\%) & 400 \\
\hline
\end{tabular}

Table II. Measured values of depth of $d_{\max }, d_{90}$ and $d_{80}$ from the PDDs for the grid-collimated electron beams with TCR, cerrobend (7) and TFP (10) grid collimators.

\begin{tabular}{lccc}
\hline Grid-collimated electron beams with: & \multicolumn{3}{c}{$\begin{array}{c}\text { Depth of grid electron } \\
\text { beams }(\mathrm{cm})\end{array}$} \\
\cline { 2 - 4 } & $\mathrm{d}_{\max }$ & $\mathrm{d}_{90}$ & $\mathrm{~d}_{80}$ \\
\hline None & 1.9 & 2.5 & 3.0 \\
TCR grid $1 \mathrm{~mm}$ & 1.2 & 1.9 & 2.4 \\
TCR grid $2 \mathrm{~mm}$ & 1.1 & 1.8 & 2.3 \\
TCR grid $3 \mathrm{~mm}$ & 0.7 & 1.5 & 2.2 \\
Cerrobend grid $1.4 \mathrm{~cm}$ (previous report) & 1.1 & 2.2 & 2.6 \\
TFP grid $5.2 \mathrm{~mm}$ (previous report) & 1.1 & 1.9 & 2.3 \\
\hline
\end{tabular}

TCR: Tungsten-contained rubber; TFP: tungsten-functional paper.

\section{Materials and Methods}

The dosimetric characteristics of the TCR grid collimators in electron grid therapy were evaluated with phantom experiments. Table I shows the physical properties of TCR. The elemental composition of TCR (mol\%) is $\mathrm{H}: 1.0 \%, \mathrm{C}: 6.5 \%, \mathrm{O}: 0.5 \%, \mathrm{~W}$ : $90.0 \%$ and others: $2.0 \%$. TCR grid collimator was created according to previous studies $(7,10)$, the aperture diameter of the holes were $2.5 \mathrm{~cm}$ and the center-to-center spacing of the holes were $3.0 \mathrm{~cm}$. The schema of the TCR grid collimator is shown in Figure 1.

The geometry for the measurements of the depth and lateral dose profiles with the TCR grid collimator is shown in Figure 2. An electron beam of a nominal energy of $9 \mathrm{MeV}$ was generated with a linear accelerator (Clinac 21EX ${ }^{\circledR}$, Varian Medical Systems, Palo Alto, CA, USA) with a $20 \times 20 \mathrm{~cm}^{2}$ electron applicator. Each measurement was taken with 200 MU irradiation. The electron beams were collimated with the TCR grid collimator placed on the surface of a virtual water phantom (MedTec Inc., Orange City, IA, USA). The thicknesses of the TCR grid collimators were 1,2 and $3 \mathrm{~mm}$. The percentage depth dose (PDD) at the center of the beam axis and the lateral dose profile (source-to-surface distance $=100 \mathrm{~cm}$ ) for the gridcollimated electron beams were measured with Gafchromic EBT3 films (ISP, Wayne, NJ, USA). The films were arranged parallel to the beam's central axis for PDD and lateral dose profile measurements.

First, the depths of the maximum dose $\left(d_{\max }\right), 90 \%$ dose $\left(d_{90}\right)$ and $80 \%$ dose $\left(d_{80}\right)$ in the virtual water phantom were evaluated from PDD data. Then, the lateral dose profiles were evaluated at $d_{\max }, d_{90}$ and $d_{80}$ with valley-to-peak ratios, defined as dose ratios of the blocked to the open areas. The valley-to-peak ratios were

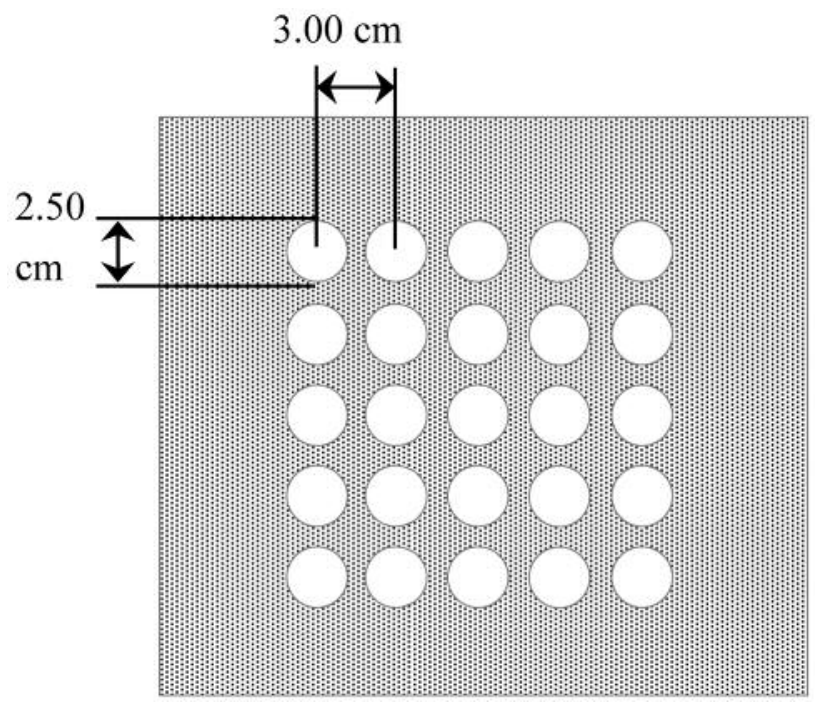

Figure 1. Schema of the TCR grid collimator. The aperture diameter was $2.50 \mathrm{~cm}$, the center-to-center spacing of holes was at $3.00 \mathrm{~cm}$ intervals and the blocked area was $80 \%$.

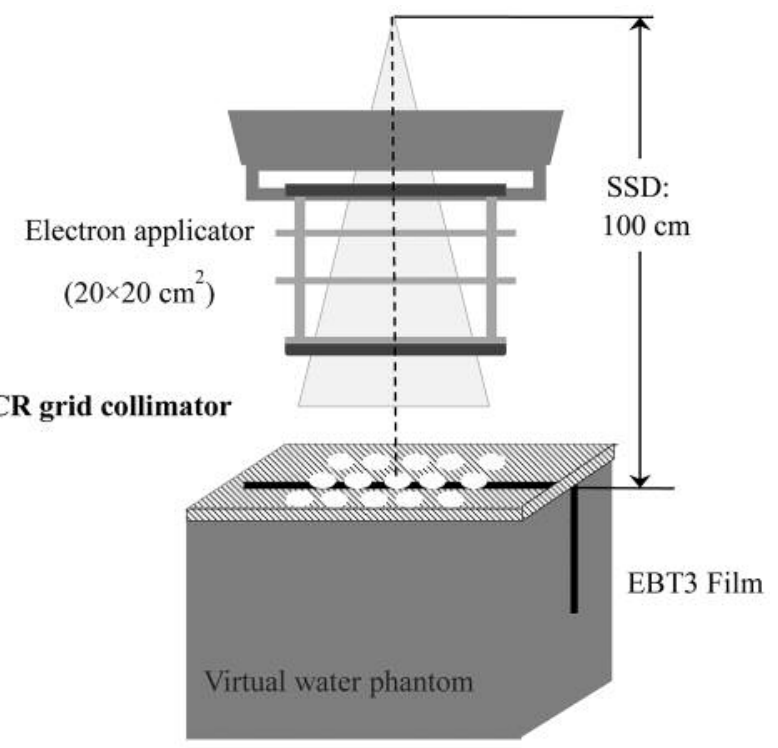

Figure 2. Schematic diagram of the experimental setup for film dosimetry in virtual water phantom. Films were arranged parallel to the beam's central axis.

calculated from the ratios of the average values at the center of four blocked areas and five open areas (10).

\section{Results}

The PDD curves for the grid-collimated electron beams at each thickness of the TCR grid collimator are shown in Figure 3. From the PDD curves, the depths of $d_{\max }, d_{90}$ and $d_{80}$ at each thickness of the TCR grid collimator are shown 


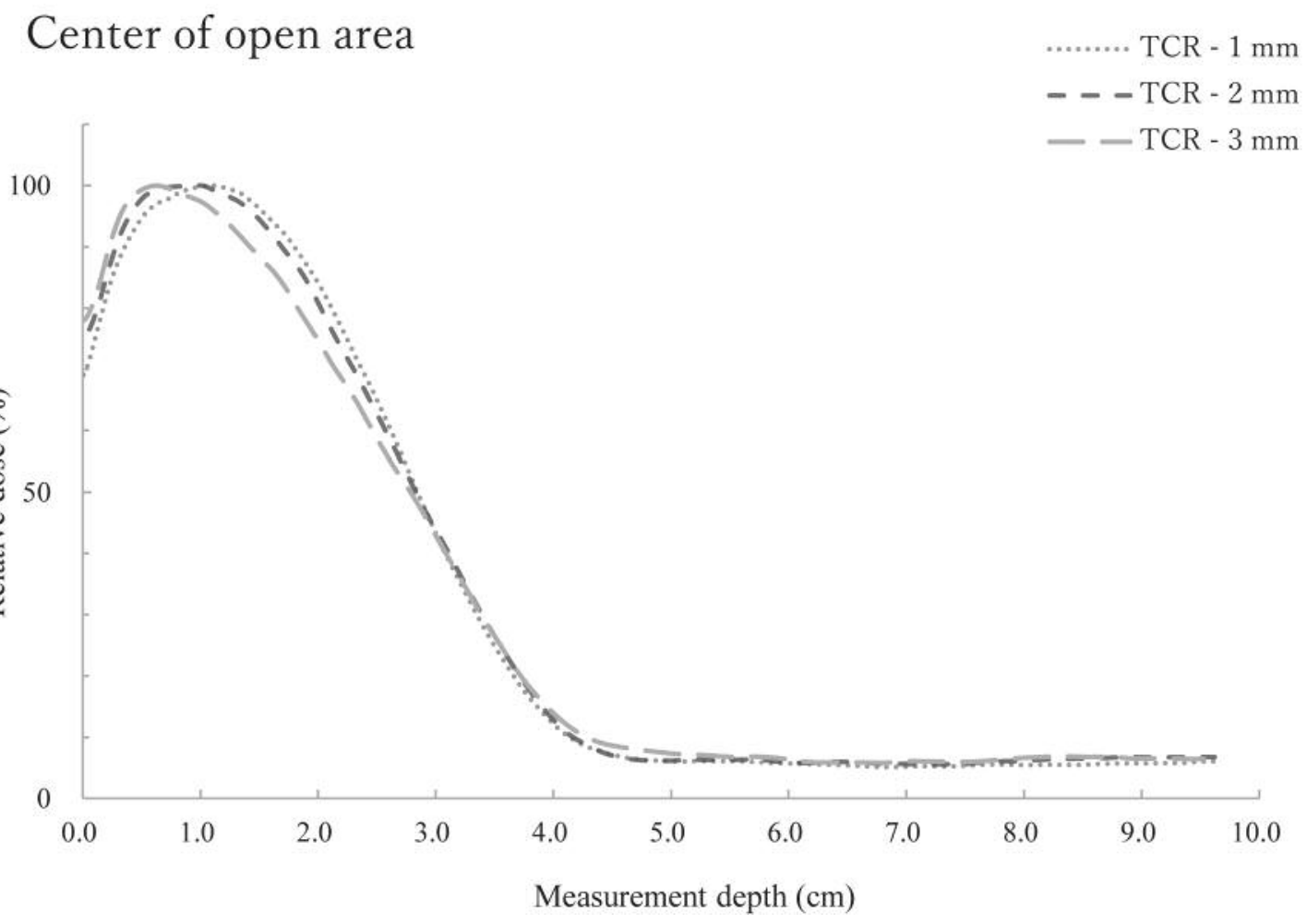

Figure 3. PDDs for the grid-collimated electron beams at $9 \mathrm{MeV}$ electron beams at each thickness of the TCR grid collimator at the center of an open area in the position of the center opening in the TCR grid collimator. The PDD curves were normalized by the value of each $d_{m a x}$.

Table III. Measured values of peaks, valleys and valley-to-peak ratios at $d_{\max }, d_{90}$ and $d_{80}$ in lateral dose profiles for the grid-collimated electron beams with TCR, cerrobend (7) and TFP (10) grid collimators.

\begin{tabular}{|c|c|c|c|c|c|c|c|c|c|}
\hline \multirow[t]{2}{*}{ Grid-collimated electron beams with: } & \multicolumn{3}{|c|}{$d_{\max }$} & \multicolumn{3}{|c|}{$d_{90}$} & \multicolumn{3}{|c|}{$d_{80}$} \\
\hline & Peak & Valley & Ratio & Peak & Valley & Ratio & Peak & Valley & Ratio \\
\hline TCR grid $1 \mathrm{~mm}$ & 100.85 & 57.06 & 0.566 & 90.02 & 59.59 & 0.662 & 79.96 & 56.40 & 0.705 \\
\hline TCR grid $2 \mathrm{~mm}$ & 99.49 & 40.95 & 0.412 & 88.40 & 48.11 & 0.544 & 79.19 & 48.10 & 0.607 \\
\hline TCR grid $3 \mathrm{~mm}$ & 100.16 & 29.32 & 0.293 & 88.63 & 43.51 & 0.491 & 80.60 & 45.59 & 0.566 \\
\hline Cerrobend grid $1.4 \mathrm{~cm}$ (previous report) & 99.63 & 47.66 & 0.478 & 87.56 & 57.72 & 0.659 & 79.84 & 58.24 & 0.729 \\
\hline TFP grid $5.2 \mathrm{~mm}$ (previous report) & 100.79 & 48.98 & 0.486 & 90.66 & 56.62 & 0.625 & 80.48 & 56.74 & 0.705 \\
\hline
\end{tabular}

TCR: Tungsten-contained rubber; TFP: tungsten-functional paper; Peak: mean relative dose at center of open area (\%); Valley: mean relative dose at center of blocked area (\%); Ratio: valley-to-peak ratios.

in Table II. The $d_{\max }$ was shifted toward the surface with increasing thickness of the TCR grid collimator. The PDD curves with the TCR grid collimator were shifted toward the surface compared to that without the TCR grid collimator.

Lateral dose profiles for the grid-collimated electron beams at each TCR grid collimator thickness at $d_{\text {max }}, d_{90}$ and $d_{80}$ are shown in Figure 4 . The mean relative doses to the open areas (as peak) and the blocked areas (as valley) and valley-to-peak ratios at $d_{\text {max }}, d_{90}$ and $d_{80}$ at each TCR grid collimator thickness are shown in Table III. The valley-to-peak ratios were increased with increasing depth, and decreased with increasing grid collimator thickness because of shielding intensity. The low valley-to-peak ratios show the large difference between the valley and peak dose values, which mean high sparing of normal tissue (10). The TCR grid collimator thickness affected the sparing effect and it was decreased with depth increasing. Therefore, the relation between the effect of sparing normal tissue and treatment range such as $d_{90}$ or $d_{80}$ was a tradeoff (10). 

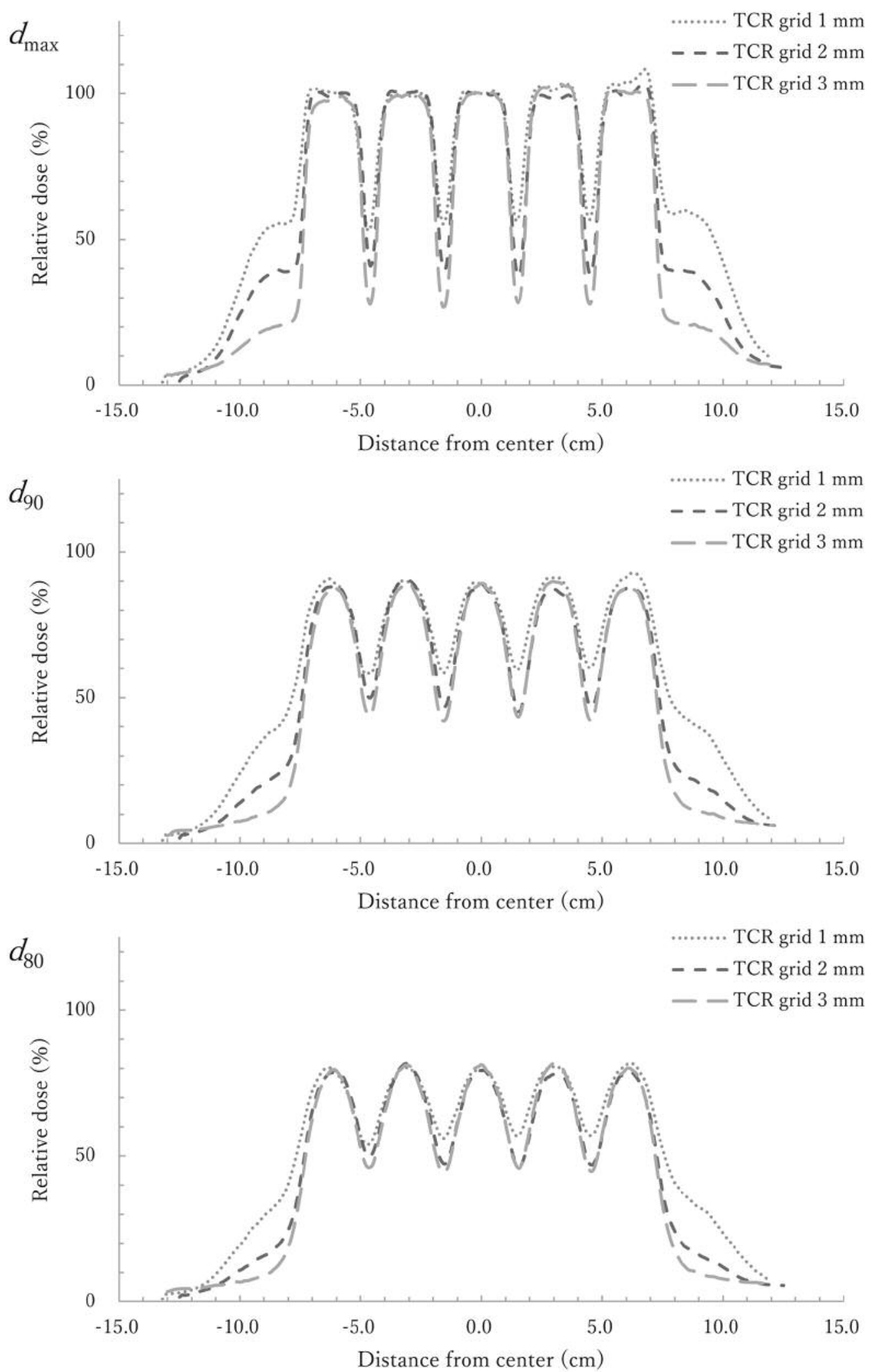

Figure 4. Lateral dose profiles for the grid-collimated electron beams at 9 MeV electron beams with TCR grid collimator of $1 \mathrm{~mm}$, $2 \mathrm{~mm}$ and $3 \mathrm{~mm}$ thickness in depths of $d_{\max }, d_{90}$ and $d_{80}$. The profiles were normalized by the central axis measurement value at the $d_{\text {max }}$ corresponding to TCR grid collimator thickness. 
As shown in Tables II and III, only the $2 \mathrm{~mm}$ TCR grid collimator achieved the equivalent dosimetric properties of a $5.2 \mathrm{~mm}$ TFP grid collimator (10) for PDD and lateral dose profiles at $d_{\max }, d_{90}$ and $d_{80}$. In addition, it also achieved the dosimetric characteristics of a cerrobend grid collimator for lateral dose profile, although the PDD curve with the TCR grid collimator was shifted to the surface compared to that with the cerrobend grid collimator, which had the same result as the TFP grid collimator.

\section{Discussion}

In this study, we showed the TCR grid collimator could be employed to the electron grid therapy by a phantom study The $2 \mathrm{~mm}$ TCR grid collimator had almost identical dosimetric characteristics as grid collimators compared to 1.4 $\mathrm{cm}$ cerrobend or $5.2 \mathrm{~mm} \operatorname{TFP}(7,10)$.

The result of PDD shows that the depths of $d_{\max }, d_{90}$ and $d_{80}$ were shifted toward the surface as the thickness of the TCR grid collimator was increasing.

The valley-to-peak ratios were changed with measurement depth, and their maximum values were at $d_{\max }$. It has been shown that valley-to-peak ratios, beam spacing and valley doses are related to radiobiological response in animal experiments (21). Wide beam spacing, low valley-to-peak ratios and low valley doses have been related to healthy tissue conservation (21). In addition, high valley-to-peak ratios without high valley doses and cold spots have been related to tumor control (21, 22 ). Based on that, the $2 \mathrm{~mm}$ TCR grid collimator was the most suitable because the peak doses, valley doses and valley-to-peak ratios were similar to previous studies $(7,10)$. To reduce the dose of irradiation field's penumbra of $2 \mathrm{~mm}$ TCR grid collimator, an additional shield could be placed up-stream of TCR grid collimator as has been shown by Tamura et al. (10).

Mohiuddin et al. have shown the usefulness of photon grid therapy for treatment of sarcoma (total response rate of 83\%) and squamous cell carcinoma (total response rate of 94\%) (4). On the other hand, photon grid therapy has not adapted to treat superficial cancers like melanoma (total response rate of 50\%) (4). Electron grid therapy was advantageous against superficial cancers as described by Meigooni et al. (7) and Tamura et al. (10). Additionally, the TCR gird collimator was better adapted to put on the patient's body surface than the TFP grid collimator owing to its flexibility, waterproof property and moderate weight. From these reasons, the TCR grid collimator was expected as excellent grid collimator to treat superficial cancers with electron grid therapy rather than cerrobend or TFP grid collimator.

\section{Conclusion}

Only the $2 \mathrm{~mm}$ TCR grid collimator, laying simply on a patient's skin, had adequate dosimetric characteristics with conventional grid collimators in electron grid therapy. The
TCR grid collimator has the features of flexibility, waterproof and moderate weight, therefore it is expected to be used for electron grid therapy instead of the conventional grid collimator.

\section{Conflicts of Interest}

Hajime Monzen received a research donation from Hayakawa rubber Co., LTD.

\section{Authors' Contributions}

KK, AK, MT and HM designed the study and the analysis. KK and AK were responsible for data collection and data management. KK, MT and HM performed data analysis. All Authors collaborated on writing the manuscript, read and approved the final manuscript.

\section{Acknowledgements}

The Authors acknowledge with thanks the staff of Division of Radiation Therapy, King Chulalongkorn Memorial Hospital, for providing the suitable equipment to validate the application of the TCR grid collimator in experiments.

This work was supported partly by JSPS KAKENHI grant numbers 16K09027, 19K17213 and 19K08211.

\section{References}

1 Laissue JA, Blattmann H and Slatkin DN: Alban Köhler (18741947): Erfinder der Gittertherapie. Z Med Phys 22: 90-99, 2012. DOI: $10.1016 /$ j.zemedi.2011.07.002

2 Jolles B: The study of connective-tissue reaction to radiation. The sieve or chess method. Br J Cancer 3: 27-31, 1949. PMID: 18140404. DOI: $10.1038 /$ bjc. 1949.3

3 Mohiuddin M, Stevens JH, Reiff JE, Huq MS and Suntharalingam N: Spatially fractionated (GRID) radiation for palliative treatment of advanced cancer. Radiat Oncol Investig 4: 41-47, 1996. DOI: 10.1002/(SICI)1520-6823(1996)4: 1<41:: AID-ROI7>3.0.CO;2-M

4 Mohiuddin M, Fujita M, Regine WF, Megooni AS, Ibbott GS and Ahmed MM: High-dose spatially-fractionated radiation (GRID): a new paradigm in the management of advanced cancers. Int J Radiat Oncol 45: 721-727, 1999. PMID: 10524428. DOI: 10.1016/S0360-3016(99)00170-4

5 Neuner G, Mohiuddin MM, Vander Walde N, Goloubeva O, Ha J, Yu CX and Regine WF: High-dose spatially fractionated GRID radiation therapy (SFGRT): A comparison of treatment outcomes with Cerrobend vs. MLC SFGRT. Int J Radiat Oncol Biol Phys 82: 1642-1649, 2012. PMID: 21531514. DOI: 10.1016/j.ijrobp.2011.01.065

6 Ha JK, Zhang G, Naqvi SA, Regine WF and Yu CX: Feasibility of delivering grid therapy using a multileaf collimator. Med Phys 33: 76-82, 2006. PMID: 16485412. DOI: 10.1118/1.2140116

7 Meigooni AS, Parker SA, Zheng J, Kalbaugh KJ, Regine WF and Mohiuddin M: Dosimetric characteristics with spatial fractionation using electron grid therapy. Med Dosim 27: 37-42, 2002. PMID: 12019964. DOI: 10.1016/S0958-3947(02)00086-9 
8 Khan FM and Gibbons JP: Khan's The Physics of Radiation Therapy. 5th ed. Philadelphia, United States, Lippincott Williams and Wilkins, 2014.

9 Lin KH, Huang CY, Lin JP and Chu TC: Surface dose with grids in electron beam radiation therapy. Appl Radiat Isot 56: 477-484, 2002. PMID: 11922415. DOI: 10.1016/S0969-8043(01)00249-4

10 Tamura M, Monzen H, Kubo K, Hirata M and Nishimura Y: Feasibility of tungsten functional paper in electron grid therapy: A Monte Carlo study. Phys Med Biol 62: 878-889, 2017. DOI: 10.1088/1361-6560/62/3/878

11 Monzen H, Tamura M, Hanaoka K, Matsumoto K and Hayakawa M: Development and application of radiation shielding paper. Ionaizing Radiat 41: 139-143, 2016.

12 Monzen H, Kanno I, Fujimoto T and Hiraoka M: Estimation of the shielding ability of a tungsten functional paper for diagnostic x-rays and gamma rays. J Appl Clin Med Phys 18: 325-329, 2017. PMID: 28656739. DOI: $10.1002 / \mathrm{acm} 2.12122$

13 Monzen H, Tamura M, Shimomura K, Onishi Y, Nakayama S, Fujimoto T, Matsumoto K, Hanaoka K and Kamomae T: A novel radiation protection device based on tungsten functional paper for application in interventional radiology. J Appl Clin Med Phys 18: 215-220, 2017. PMID: 28422397. DOI: 10.1002/acm2.12083

14 Fujimoto T, Monzen H, Nakata M, Okada T, Yano S, Takakura T, Kuwahara J, Sasaki M, Higashimura K and Hiraoka M: Dosimetric shield evaluation with tungsten sheet in 4, 6, and 9MeV electron beams. Phys Medica 30: 838-842, 2014. PMID: 24953537. DOI: 10.1016/j.ejmp.2014.05.009

15 Kamomae T, Monzen H, Kawamura M, Okudaira K, Nakaya T, Mukoyama T, Miyake Y, Ishihara Y, Itoh Y and Naganawa S: Dosimetric feasibility of using tungsten-based functional paper for flexible chest wall protectors in intraoperative electron radiotherapy for breast cancer. Phys Med Biol 63: 015006, 2017. PMID: 29083315. DOI: 10.1088/1361-6560/aa96cf

16 Kosaka H, Monzen H, Matsumoto K, Tamura M and Nishimura Y: Reduction of operator hand exposure in interventional radiology with a novel finger sack using tungsten-containing rubber. Health Phys 116: 625-630, 2019. DOI: 10.1097/ HP.0000000000000992
17 Kijima K, Monzen H, Matsumoto K, Tamura M and Nishimura Y: The shielding ability of novel tungsten rubber against the electron beam for clinical use in radiation therapy. Anticancer Res 38: 3919-3927, 2018. PMID: 29970513. DOI: 10.21873/ anticanres. 12677

18 World Health Organization(WHO). Environmental Health Criteria165, inorganic lead., 1995. Available from: http:// www.inchem.org/documents/ehc/ehc/ehc165.htm

19 World Health Organization(WHO). Safety Evaluation of Certain Food Additives and Contaminants, WHO Food Additive Series: 44, 2000. Available from: http://www.inchem.org/ documents/ jecfa/jecmono/v44jec12.htm

20 Rapisarda V, Ledda C, Castaing M, Proietti L and Ferrante M: Potential exposure to carcinogens in low-melting alloys processing. G Ital Med Lav Ergon 35: 73-76, 2013. PMID: 23914599.

21 Dilmanian FA, Button TM, Le Duc G, Zhong N, Pena LA, Smith JAL, Martinez SR, Bacarian T, Tammam J, Ren B, Farmer PM, Kalef-Ezra J, Micca PL, Nawrocky MM, Niederer JA, Recksiek FP, Fuchs A and Rosen EM: Response of rat intracranial 9L gliosarcoma to microbeam radiation therapy. Neuro Oncol 4: 26-38, 2002. PMID: 11772430. DOI: 10.1093/ neuonc/4.1.26

22 Regnard P, Duc G Le, Bräuer-Krisch E, Troprès I, Siegbahn EA, Kusak A, Clair C, Bernard H, Dallery D, Laissue JA and Bravin A: Irradiation of intracerebral 9L gliosarcoma by a single array of microplanar x-ray beams from a synchrotron: Balance between curing and sparing. Phys Med Biol 53: 861-878, 2008. DOI: $10.1088 / 0031-9155 / 53 / 4 / 003$ 